\title{
Online Design Discussion Sites: Emerging Resource for Creative Design
}

\author{
Moushumi Sharmin and Brian P. Bailey \\ University of Illinois at Urbana-Champaign \\ Department of Computer Science \\ Urbana, IL, USA \\ \{sharmin2, bpbailey\} aillinois.edu
}

\begin{abstract}
Online design discussion sites are a popular networking platform drawing thousands of designers from all around the globe. These sites show promise as emerging design resource by enabling designers to learn about design techniques and tools, share ideas and to seek (receive) critique to help refine their ideas, and to learn from other designers' experience. In this paper, we report results of a study that aims to understand designers' experience and activities in online design discussion site and how they relate to design. We have analyzed member profiles $(\mathrm{N}=12164)$, posts submitted by one-time posters $(\mathrm{N}=2854)$, and post-contents $(\mathrm{N}=1004)$ for a month and conducted follow-up interviews $(\mathrm{N}=5)$ to investigate what roles such sites play in supporting design. Our findings reveal that while designers find such sites extremely promising, ineffective representation of ongoing activities, lack of visibility of contribution, and difficulty in finding needed information hinder (even discourage) participation. We propose actionable implications that can lead into better site design.
\end{abstract}

Keywords: Creative Design, Design Community, Online Design Discussion Site, Design Critique.

\section{Introduction}

Online discussion sites provide a place for informal conversation for people with similar experience [1] as well as offer opportunities for building and maintaining relationships. Online discussion sites serve as effective sources of communication [2], information gathering and entertainment [3]. Online design discussion sites (ODDS) are a small but important subset, attracting many designers especially students and freelancers - offering an informal environment for communication and collaboration.

Design researchers consider creative design to be a social process [4, 5] and creative outcomes are attributed to the discussion and collaboration among designers as it promotes divergent thinking [6]. ODDS thus show promise in supporting design creativity by allowing designers to share and receive critique on their ideas from a diverse group of designers, making design sociable. For many freelance and student designers who don't have access to a design team or colleagues to assist in the design process, ODDS are the only source of critique. 
Due to the popularity of the online networking and socializing sites, a large number of researchers have focused on understanding collaborative content-creation [7, 8, 9] and users' socializing behavior in this setting [10]. In addition to the facilities that any online discussion site may provide, ODDS present an opportunity to share early ideas and receive critique from a diverse group of designers - allowing the designer to consider different perspectives and thus making these sites valuable resource for creative design. We believe research focusing on understanding designers' experience and activities in ODDS and how these ongoing activities relate to design will lead to better design support sites - enhancing the utilization of ODDS as a resource for creative design. We extend research in this area by taking a first step towards this goal - aiming to offer insight about current practices and to identify areas that need to be addressed to increase the effectiveness of such sites.

In this paper, we report results from a study that investigated activities, contents, and user experience in a popular ODDS aiming to understand the role of such sites as a design resource. Our study offered several important insights - (1) requests for critique and information about design techniques account for $70 \%$ of the total activity; (2) novice (mainly student) designers utilize these sites to seek critique and to learn about design techniques and tools; (3) expert designers (mainly freelance) utilize these sites to find collaborators and to promote their work; (4) critique and information requests receive notable number of responses within a short period of time (on an average, 7.8 and 5 replies respectively within the same day); (5) contribution is highly skewed with only $6.1 \%$ members contributing $83.28 \%$ of total content; (6) contribution is a function of members' need, rank, and experience with the site; and (7) members' experience varies as a function of involvement with the site and the information provided on their profiles. We also offer actionable implications that can improve the design of ODDS.

\section{Related Work}

Internet facilitates information sharing using various systems including online networks [11]. Online discussion sites are successors of online bulletin boards which created the culture of mass communication $[1,12]$. Bulletin boards and early discussion sites concentrated on information sharing and problem solving in specific domains such as sports, politics, religion, technical hot lines, etc. With the rapid increase of Internet users, discussion sites became a part of the online culture [13].

Recent years have seen tremendous growth in the popularity of online design discussion communities (ODDS) (e.g., core77, highend3d, about.com). ODDS show potential to support creativity in design by providing a platform for exchanging ideas, refining ideas based on critique from a diverse group of designers through open communication and collaboration. However, ODDS look exactly like any other discussion group and provide little support for identifying the ongoing design activities - hindering the effective utilization of these sites as a design resource. Little is known about the designers' needs and experience surrounding ODDS. In our research we take a first step towards filling this gap by offering insight about existing practice and identifying areas that deserve attention. 
Researchers examined the pattern of participation in the online setting, especially sites that create encyclopedic material [9], provide entertainment resources [7, 8], and/or offer social networking capabilities [10]. Work on online movie databases examined factors that influence contribution [7]. Considerable amount of work also focused on the contribution pattern in Wikipedia $[14,15,9]$. While both entertainment and content-creation communities try to motivate users to contribute by creating an opportunity for self-satisfaction, ODDS additionally offer an opportunity to enhance the quality of designs by receiving critique on ongoing designs from a diverse group of designers. The difference in ongoing activities and users' expectation makes it difficult to directly apply lessons from other communities to encourage effective participation in design discussion sites. Our work attempts to extend existing research on online communities by offering deeper understanding about ongoing activities and factors that influence designers' experience in these sites.

\section{Methodology}

The purpose of our research was to gain insight about activities and motivations for participation in ODDS and whether and how they relate to design. Our study combined both quantitative and qualitative research methods to investigate the activities and learn about user experience of core77, a thriving online design discussion site [16]. For our study, we have chosen core77, but we believe that our findings are generalizable to other ODDS.

We have collected and analyzed member profiles $(\mathrm{N}=12164)$ along with post history (rank, number of posts, number of replies) to examine participation pattern and to identify factors that influence members' experience in the site. We have also analyzed all contents posted in a month $(\mathrm{N}=1004,177$ threads receiving 827 replies) to investigate types of posts and their relation to different design activity. Additionally, we have analyzed all posts $(\mathrm{N}=2854)$ submitted by the one-time posters (members who posted just once) to identify underlying factors, if any, that motivated the only post but hindered or discouraged further active participation. To better understand members' experience, we have conducted semi-structured interviews with five core77 users who have varying level of involvement with the site - ranging from administrator to semi-active users. See table 1 for a sample of the interview questions.

Table 1. Sample of the questions asked during the interview

\begin{tabular}{|l|}
\hline Participation \\
\hline What factors influence your participation in the site? \\
\hline Does your participation pattern change with time? What factors influence this change? \\
\hline Motivation \\
\hline What motivates you to contribute in the site? \\
\hline From your perspective, what are the potential benefits of active participation in the site? \\
\hline Challenge \\
\hline From your perspective, what are the main obstacles that hinder active participation? \\
\hline
\end{tabular}




\section{Study Results}

\subsection{Post Categories - Conversational, Design Technique and Information, Informational, and Critique}

Designers participate in ODDS for a variety of reasons, including informal conservation, query about design tools and techniques, seeking critique about ongoing ideas or just sharing information about design events. In core 77 , there are 21 different topic categories from which we identified 12 most active categories, such as general design discussion, sketching, portfolio, etc. in terms of number of posts and views. Analysis of the posts indicated that posts related to critique and design technique dominate 10 of the 12 most active categories. Overall, requests for critique and question regarding design techniques and tools accounted for over $70 \%$ of the posts in the 12 category studied. This clearly indicates the predominant utilization of these sites as a resource for creative design as opposed to a place for informal networking.

We have analyzed the post-contents to identify types of posts and whether and how they relate to different types of design activity. Content analysis revealed that posts can be broadly categorized into four types - (i) Conversational: Informal experience sharing - targeted to initiate discussion (e.g., good examples of "design language"); (ii) Design Technique and Information: Query seeking information on tools and techniques (e.g., How do I do good transition surfaces?); (iii) Informational: Posts containing links of design events, jobs, blogs, or articles (e.g., design job websites); and (iv) Critique: Posts seeking or providing critique on a design, often including a link to the project or portfolio or images along with a brief description of the project (e.g., feedback please...maybe a critique here and there). Figure 1 presents a screen capture of one such category indicating that different types of posts get represented in the same manner providing no information about the type or content.

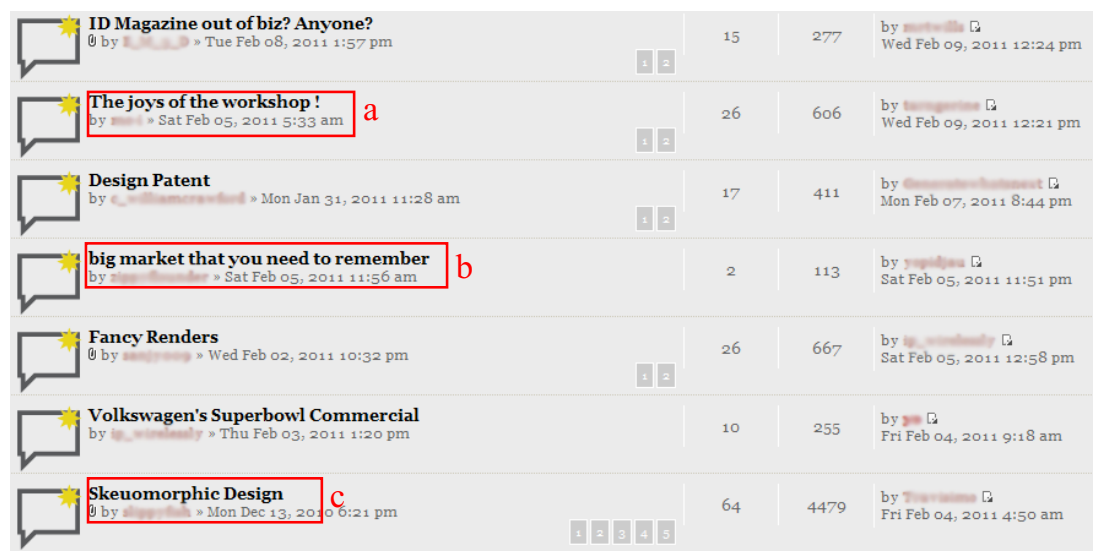

Fig. 1. Posts from one category of core77. Posts can be categorized as a) conversational; b) informational; and c) design techniques and tools, but the current interface represents all these similarly, providing no feedback to the members about the post types. 
Table 2. Post categories and activities surrounding posts by one-time posters in core77

\begin{tabular}{|l|l|l|l|l|}
\hline Category & Average (Reply) & Stdv (Reply) & Average (View) & Stdv (View) \\
\hline Design Tech. and Info. & 5.06 & 10.58 & 2210.26 & 4584.07 \\
\hline Conversational & 12.23 & 18.86 & 5458.22 & 13570.98 \\
\hline Critique & 7.82 & 7.90 & 2844.88 & 2620.85 \\
\hline Informational & 1.58 & 2.48 & 1168.11 & 1009.13 \\
\hline
\end{tabular}

Our interviewees expressed that not all designers are interested in all types of posts and consider it difficult to find posts of interest. Similar finding has also been reported in [17] for online Q and A sites, where some users preferred to look at informational vs. conversational posts. The high cost associated with finding posts that demand attention and can be benefitted by a members' response limits the utilization of such sites. As a result, to ensure effective participation, it is utmost important to distinguish between different types of posts. While categorization and filtering of posts based of types will allow the members to find posts of interest effectively, categorization solely based on content is difficult and often imperfect as the original and subsequent posts can fall into multiple categories. Providing option for indicating the post type along with content-based categorization may lead to better representation of posts.

\subsection{Pattern of Participation: A Function of Need, Rank, and Expertise}

Posts are prompted to satisfy information need: $75 \%$ of one-time posters posted on the same day they have become registered members. This behavior may occur due to the fact that users are "required to register" to post. $60 \%$ of the one-time posters were seeking information on specific design tool or technique while $28.6 \%$ engaged in conversational topics. Interestingly, only $5.6 \%$ of the one-time posters were requesting critique - emphasizing members' tendency to become familiar with the site before posting a critique request. See table 2 for the community response for posts submitted by one-time posters - reflecting that even new members receive significant number of replies and views from the community. Interestingly, $30 \%$ of these one-time posts also replied to other designers' posts, indicating the presence of passive participation from a significantly large number of users.

Ranking as an ineffective mechanism to encourage contribution: Like many existing online communities, Core77 uses a ranking model to categorize its members. The current ranking model utilized is as follows: Administrator, Moderator, Full-self Realization, Step four, Step three, Step two, Step one, and then members without any rank. See table 3 for a rank-based distribution of members and their contribution. Moderators and administrators not only maintaining a clean and welcoming environment but also are the most active users (23\% posts are contributed by them who are $0.13 \%$ of the total members). Most of the other members take a semi-active role, posting when needed. Positions such as moderators and administrators are usually awarded to members who have high rank (calculated based on number of posts). While ranking is used as a mechanism for encouraging participation, the current ranking is proved ineffective as $93 \%$ users in the site have no rank, with $39.5 \%$ never posting anything and an additional $23.5 \%$ posting just once. High effort required for finding thread of interest is reported as one of the main reasons hindering contribution. 
Table 3. Contribution statistics of members in core 77

\begin{tabular}{|l|l|l|c|l|c|c|}
\hline Rank Category & $\begin{array}{l}\text { Post } \\
\text { (Required) }\end{array}$ & $\begin{array}{l}\text { Post } \\
\text { (Avg.) }\end{array}$ & $\begin{array}{l}\text { Member } \\
(\% \text { of } \\
\text { Total) }\end{array}$ & $\begin{array}{l}\text { Post } \\
(\% \text { of } \\
\text { Total })\end{array}$ & $\begin{array}{l}\text { Member } \\
\text { (cum \%) }\end{array}$ & $\begin{array}{l}\text { Post } \\
\text { (cum \%) }\end{array}$ \\
\hline Administrator & - & 3275.0 & 0.02 & 7.35 & 0.02 & 7.35 \\
\hline Moderator & - & 1608.3 & 0.11 & 15.6 & 0.13 & 22.95 \\
\hline $\begin{array}{l}\text { Full self- } \\
\text { realization }\end{array}$ & $>=600$ & 943.1 & 0.19 & 16.2 & 0.32 & 39.15 \\
\hline Step four & $200 \sim 599$ & 340.6 & 0.57 & 17.6 & $\mathbf{0 . 8 9}$ & $\mathbf{5 6 . 7 5}$ \\
\hline Step three & $100 \sim 199$ & 136.9 & 0.75 & 9.3 & 1.64 & 66.05 \\
\hline Step two & $50 \sim 99$ & 68.7 & 1.40 & 8.7 & 3.04 & 74.75 \\
\hline Step one & $20 \sim 49$ & 30.2 & 3.06 & 8.4 & $\mathbf{6 . 1}$ & $\mathbf{8 3 . 1 5}$ \\
\hline No rank & $<20$ & $\mathbf{2 . 0}$ & $\mathbf{8 9 . 5}$ & $\mathbf{1 6 . 7}$ & 95.6 & 99.85 \\
\hline Spammer & - & 0.00 & 3.19 & 0.00 & 98.8 & \\
\hline Banned & - & 0.19 & 1.2 & 0.02 & 100 & \\
\hline
\end{tabular}

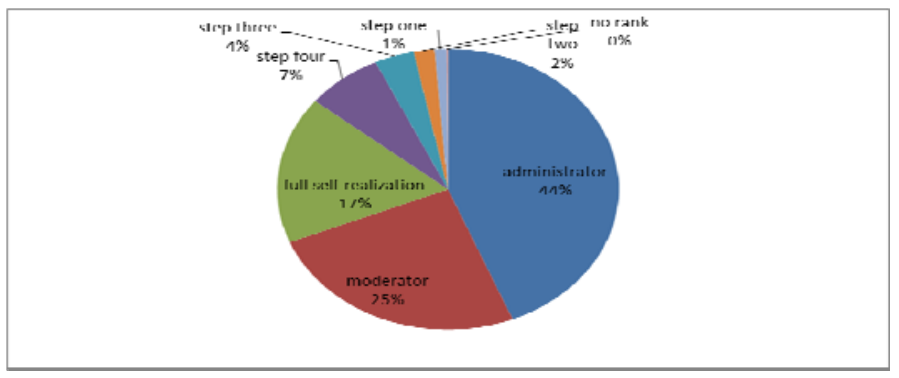

Fig. 2. Distribution of rank-wise daily posts

Time of involvement with the site and expertise influences participation: In core77, $93 \%$ of all daily posts are replies from members who are involved with the site for a long time and are highly ranked members of the community (see figure 2). While a majority of the new members post on their first day, only $0.005 \%$ of the daily post is submitted by the new members. Expertise of the members also plays an important role in the participation. In core77, expert designers rarely request critique on their own design, but offer critique and/or information on design to novice members. While novice designers mostly seek help, expert designers almost always offer help. Interview revealed that expert designers utilize other medium for seeking critique - mostly sending e-mails to friends whom they found and build relationship through the site. Expert designers also consider providing critique as a way of repaying the design community. The quotes from two interviewees nicely summarize this behavior:

"If designers are just going into the industry, I feel more apt to share information with them as I don't want them to fall into the same holes where I fall on to...I try to respond to requests for design feedback as much as possible." 
"I started using core77 as a student and I was blown away with the idea of being able to interact with real designers. I like the fact that as a professional, I am accessible to students and can give back what I've earned over the past decade in a way that is easy to access and fully documented."

\subsection{Motivation for Participation}

Finding mentors and collaborators: Student and freelance designers, who have limited access to expert designers, utilize ODDS to contact expert designers in their area of design. The site provides opportunity to create relationship with expert designers and find mentors and collaborators in an informal environment. Freelance designers often mention ODDS as the place for meeting other designers, finding mentors and collaborators for future projects. However, while long-time members find mentors or collaborators by judging the interaction history, new comers in a site have no information about how to search for a potential mentor/collaborator. One designer commented:

"If you are looking at sites, where you see people again and again, it's the perception that you grow about the people, what type of materials they post, how is the quality of information, how do they help, etc. to see how he will be able to mentor me personally. Ask questions and see how the designers respond. (But) background and expertise information about designers would be great as then there will be experts to whom you can go to."

Access to design resources: Members utilize the site to receive critique from a diverse group of designers. Additionally, the site features articles and events of latest and greatest happenings in the world of design. Designers view it as a one stop place for their informational, emotional needs, nicely summarized by a designer:

"We joined to discuss among us, but it was a useful source for getting feedback, professional people give you advice, you can get news about things that interest you, there are groups that discuss overseas jobs, recruiters also participate in this site. It is a place where you can get all sorts of news and entertainment."

Experience sharing in a less competitive environment: Designers become users of online sites, primarily to interact with designers who share similar experiences but could not be reached by any other medium. ODDS also provide an environment where designers can share ideas without the stress of constant evaluation and peer pressure, which can enhance design creativity [5]. One designer stated:

"Online forums create a much more accessible, less formal community than attending local IDSA or AIGA events where the designer is often with people he/she is directly competing with. The forum can be anonymous and less threatening."

\subsection{Influence of Profile Information on Member Activity and Experience}

While race, gender, and social status can highly impact the interaction of participants in offline communities, in online setting it is likely to have less impact. However, users' self-portrayal in an online setting influences her networking and communication behavior. Lampe et al. discussed how inclusion of certain information in user's Facebook profile enhanced their friend-making capacity [18]. In core77, members can 
add (optional) information such as website, occupation, and avatar in their profiles. The average number of replies received is 23.2 vs. 4.3 for users who provided additional info vs. no information. While there is no option for providing information on race, gender or social status, our interviewees expressed that gender and affiliation play a role to gain more attention. One designer commented:

"Better school usually gives a certain amount of respect to posters. Women are usually given special attention because they are rare (in ID at least).”

In core77-like setting, it is difficult to verify user profile and racial and gender association. In-depth analysis is needed to learn how these information impacts members' experience with the site.

\subsection{Lack of Control over Audience Forces Interaction to Be Not So Informal}

As a medium, ODDS preserve all user interaction history and it is possible to track down members responsible for posts by any other member and at any given point. This makes participants conscious about their interaction as they can be held responsible for a comment that they made 10 years ago when they were unfamiliar with the setting. This everlasting memory of the site hinders fluid participation as it is impossible to imagine target audience [14] and participants have no control over it. Allowing participants to define their own privacy model (e.g., Facebook allows users to define who can view their profile, posted materials, etc.) may encourage more designers to participate more as they will have control over their posted materials.

\section{Design Implications and Discussion}

\subsection{Create Awareness of Posts of Interest}

Though there are high level categories that guide users to post their requests under a specific topic, each of these categories contain a number of subcategories and get numerous new posts every day. The overhead of finding threads that require help and would benefit from a reply is extremely high. This leads to inefficient distribution of efforts where some posts get more responses while others get overlooked. One solution can be routing posts to different users based on their area of expertise. This routing can be done automatically by collecting data from a user's profile and by analyzing previous posts and interaction history.

\subsection{Provide Activity-Based Summary of the Categories}

Like almost all ODDS, core77 interface provides a linear list of discussion categories with an icon indicating new posts. To read a post, users have to go through a series of steps and sometimes several pages before finding anything interesting. Also, to get an idea about the post content, users have to click on the individual posts to see the original and subsequent posts. Additionally, all the posts look similar despite differences in their contents. One possible solution is to provide a visual summary (graphs indi- 
cating new posts and number of replies and views) of the activities of each category in the discussion home page - providing better awareness of ongoing activity. Highlighting keywords from new threads may also reduce the burden on users to search for topics of interest.

\subsection{Support Categorization of Posts Based on Content}

At present all posts are presented under the same label irrespective of the content. However, not all members are interested in all types of posts and categorizing posts such as conversational or critique will benefit members to effectively find posts of interest. Similar finding has been reported by [17] in context of Q and A sites. Providing a default set of tags or keywords such as conversational, critique will allow users to attach these to their posts. Alternatively, content classifiers can be used to suggest tags or keywords to a user for a new post and users can accept or select a tag that best describes the post.

\subsection{Provide Awareness of (Quality) Contribution}

Like many other sites, ranking in core 77 is solely based on number of posts - ignoring the quality and impact of the posted material (e.g., an in-depth critique is considered similar to a comment like "great work!"). The ranking system also ignores works such as efforts required to maintain a clean environment (site). Users who contribute quality materials or are engaged in the maintenance often feel demoralized as their contribution is not visible and well-recognized.

Making contribution visible will make users aware of their and other's contribution. Adding an automated activity graph in users' profile can help in this direction. To reflect the impact and quality of a contribution, users can award points or rank the post and it can be used along with number of posts to determine overall ranking. As contributions will be judged in terms of quality and quantity, users may feel motivated to contribute frequently and it may also improve the quality of contribution. Additionally, providing a summary of top contributors may motivate more users to actively participate as they become aware of the level of effort necessary to achieve higher rank in the community.

\section{Conclusion}

This paper offers insight about activities and designers' experience and participation pattern in ODDS. The study sheds light on the factors that influence particpartion in these sites - highlighting areas that need improvement. As more and more designers are getting involved in these communities every day, it is important to understand what factors hinder their participation and how we can assist in lowering these barriers. As a new form of creative community there is a lot to learn about the audience of these groups, individual's perception of privacy and security in this setting and how that can be maintained, and how individuals can best utilize this setting for enhancing design creativity. Ineffective representation of activities and information are considered as one of the major factors that hinder participation. Another major issue observed is the lack of active participation by the majority of the members. To ensure the success of such communities, it is essential that a significant number of members 
actively participate and eventually reduce the burden on the few committed ones who carry the community. In-depth research is needed to understand how to close the gap between passive and active participation and encourage more people to effectively participate. Another area of future research is the role of status and power in this setting and how that affects overall participation.

\section{References}

1. Wellman, B., Gulia, M.: The network basis of social support: A network is more than the sum of its ties (1999)

2. Joinson, A.N.: Looking at, looking up or keeping up with people? motives and use of facebook. In: Proc. CHI, pp. 1027-1036 (2008)

3. Nardi, B.A.: Beyond Bandwidth: Dimensions of Connection in Interpersonal Communication. CSCW 14(2), 91-130 (2005)

4. Cross, N., Cross, A.: Observations of Teamwork and Social Processes in Design. Design Studies 16(2), 143-170 (1995)

5. Warr, A., O’Neill, E.: Understanding design as a social creative process. In: Proc. Creativity \&Cognition, pp. 118-127 (2005)

6. Roy, R.: Case Studies of Creativity in Innovative Product Development. Design Studies 14(4), 423-443 (1993)

7. Beenen, G., et al.: Using Social Psychology to Motivate Contributions to Online Communities. In: Proc. CSCW, pp. 212-221 (2004)

8. Lange, P.G.: Publicly Private and Privately Public: Social Networking on YouTube. Computer-Mediated Communication 13(1), 361-380 (2007)

9. Viegas, F.B., Wattenberg, M., K., D.: Studying cooperation and conflict between authors with history flow visualizations. In: Proc. CHI, pp. 575-582 (2004)

10. Boyd, D.: Social Network Sites: Public, Private, or What? Knowledge Tree, 13 (2007)

11. Mislove, A., Marcon, M., Gummadi, K.P., Druschel, P., Bhattacharjee, B.: A Measurement and analysis of online social networks. In: Proc. ICM, pp. 29-42 (2007)

12. Whittaker, S., Terveen, L., Hill, W., Cherny, L.: The Dynamics of Mass Interaction. In: Proc. CSCW (1998)

13. Kollock, P., Smith, M.: Managing the Virtual Commons: Cooperation and Conflict in Computer Communities. Computer-Mediated Communication: Linguistic, Social, and Cross-Cultural Perspectives, 109-128 (1996)

14. Cosley, D., et al.: SuggestBot: using intelligent task routing to help people find work in wikipedia. In: Proc. IUI, pp. 32-41 (2007)

15. Kriplean, T., Beschastnikh, I., McDonald, D.W.: Articulations of WikiWork: Uncovering Valued Work in Wikipedia through Barnstars. In: Proc. CSCW (2008)

16. Core77 Discussion Board, http: / / boards . core77 . com/

17. Harper, M., Moy, D., Konstan, J.: Facts or friends?: distinguishing informational and conversational questions in social Q\&A sites. In: Proc. CHI, pp. 759-768 (2009)

18. Lampe, C., Ellison, N., Steinfield, C.: A familiar face(book): profile elements as signals in an online social network. In: CHI 2007, pp. 435-444 (2007) 